\title{
Effect of temperature on morphology of triethanolamine-assisted synthesized hydroxyapatite nanoparticles
}

\author{
Nagaprasad Puvvada • Pravas Kumar Panigrahi • \\ Himani Kalita • Keka R. Chakraborty • \\ Amita Pathak
}

Received: 21 December 2011/ Accepted: 5 May 2012/Published online: 24 May 2012

(C) The Author(s) 2012. This article is published with open access at Springerlink.com

\begin{abstract}
Hydroxyapatite (HA) nanoparticles have been synthesized using ortho-phosphoric acid as the source of $\mathrm{PO}_{4}{ }^{3-}$ ions, and calcium chloride, suitably complexed with triethanolamine, as the calcium source. The effect of temperature on the morphology of the product has been investigated. The chemical compositions of the samples have been established through Fourier transform infrared spectroscopy. This study reveals that A-type and B-type carbonate substitution are present in HA samples and the concentration of carbonate ions decrease with rise in temperature. Morphological analyses by TEM studies suggest that the average lengths and widths of the needle-shaped particles size increases with temperature up to $50{ }^{\circ} \mathrm{C}$, while a morphological change of the particles from needle to spherical shape is observed on raising the temperature above $50{ }^{\circ} \mathrm{C}$. This change in morphology has been assigned to the apparent solubility of $\mathrm{HA}$ at these temperatures, which has been studied by the determination of thermochemical properties of the reaction system by endpoint conductivity and electrophoretic mobility measurements.
\end{abstract}

Keywords Hydroxyapatite - Temperature - Morphology · Endpoint conductivity

\footnotetext{
N. Puvvada · P. K. Panigrahi · H. Kalita · A. Pathak ( $\square)$ Department of Chemistry, Indian Institute of Technology, Kharagpur 721302, West Bengal, India e-mail: ami@chem.iitkgp.ernet.in

K. R. Chakraborty

Variable Energy Cyclotron Centre, Kolkata 700064,

West Bengal, India
}

\section{Introduction}

Hydroxyapatite (HA) is one of the chemically and biologically important materials due to its similarity with human hard tissues, such as bone, dentine, etc. It is used as artificial bone material because of its biocompatibility, osteoconductivity and bioactivity, i.e., its ability to form direct bond with tissue (Puvvada et al. 2010; Sonju Clasen and Ruyter 1997). Due to their biocompatibility, they are promising materials in separation techniques, e.g., for the removal of numerous heavy metal ions (especially lead and cadmium ions) from waste water through ion-exchange process (Puvvada et al. 2010), and in drug delivery systems for the delivery of anti-tumor agents and antibodies in the treatment of osteomyelitis (Xia and Chang 2006; Yang et al. 2005; Yamashita et al. 1998). The properties and applications of synthetically prepared HA have been reported to be influenced by the size and morphological characteristics of their particles (Ramachandra et al. 1997). For example, smaller crystal size and more imperfect crystals, being subjected to dissolution (LeGeros 1988; Hurson et al. 1993), may affect the extent of bone loss in osteoporosis and other metabolic diseases (Glenn 2008). These size-dependent properties of HA motivated researchers to adopt a wide-range of synthesis techniques, such as chemical precipitation (Puvvada et al. 2010), hydrothermal processing (Luo and Nieh 1996), sol-gel (Shirkhanzadeh 1998), micro-emulsion processing (Earl et al. 2006), wet chemical methods incorporating a freezedrying step (Bogdanoviciene et al. 2006), and microwave irradiation (Lim et al. 1997) to obtain HA nanoparticles with varied sizes and morphologies. The wet chemical process, based on precipitation route, is however the most convenient and commonly used process for the synthesis of nanocrystalline HA material. In most of these conventional 
precipitation methods, the factors those precisely govern the precipitation, i.e., $\mathrm{pH}$, temperature, $\mathrm{CO}_{3}{ }^{2-}$ ion concentration, $\mathrm{Ca} / \mathrm{P}$ mole ratio, etc., influence the morphological changes, resulting in the formation of $\mathrm{HA}$ nanocrystals with varying dimensions (Uota et al. 2005). According to some previous reports (Ramachandra et al. 1997; Kumar et al. 2004; Prakash et al. 2006a, b), the size of HA particle increases with increase in temperature, which has been attributed to the change in the concentration of $\mathrm{Ca}^{+2}$ and $\mathrm{CO}_{3}{ }^{-2}$ ions $\mathrm{pH}$, etc. For example, Prakash et al. $(2006 \mathrm{a}, \mathrm{b})$ found that the morphology of HA nanoparticles changed from needle shape to spherical, when the reaction of the sparingly soluble salts $\left(\mathrm{Ca}(\mathrm{OH})_{2}\right.$ and $\mathrm{H}_{3} \mathrm{PO}_{4}$ ) was carried out in the temperature range 30-100 ${ }^{\circ} \mathrm{C}$. However, needle-shaped HA nanoparticles with constant increase in dimensions were produced by raising the temperature up to $100{ }^{\circ} \mathrm{C}$ and by using completely soluble salts, such as, $\mathrm{Ca}\left(\mathrm{NO}_{3}\right)_{2} /\left(\mathrm{NH}_{4}\right)_{2} \mathrm{HPO}_{4}$ or $\mathrm{CaCl}_{2} / \mathrm{Na}_{3} \mathrm{PO}_{4}$. Based on the discussion it can thus be inferred that the method of preparation, in combination with the type of reagents used and control of the experimental parameters, can influence the size and morphology, and hence, the properties of the final product. In addition, it is also important to maintain the $\mathrm{Ca} / \mathrm{P}$ mole ratio between 1.64 and 1.68 in the HA samples to obtain the most stable form of calcium phosphate (Puvvada et al. 2010). In some cases, however, the formation of other calcium phosphate by-products, such as di- and tri-calcium phosphates, was reported along with HA (Kumar et al. 2004). It is thus possible to manipulate the size, shape and composition of HA samples with desired properties through appropriate choice of the preparation route and suitable control of experimental parameters. Xu et al. (2012) reported different sizes and morphologies of nano-HAP induced significant apoptosis in osteoblast cells. In our previous study, we reported (Puvvada et al. 2012) shape-dependent magnetite nanoparticles peroxidase mimetic activity on pyrogallol substrate. Moreover, these magnetite nanoparticles with different morphologies can be implicated in biological applications as well.

Motivated by this fact, in the present work, we have prepared HA samples with varied sizes and morphologies by simply varying the reaction temperature of completely soluble salts (i.e., calcium chloride and ortho-phosphoric acid) in the presence of triethanolamine (TEA) as complexing agent through an aqueous-based chemical precipitation method. In this study, we have observed a different trend of morphology variation compared to that found by Prakash et al. (2006a, b). The change in morphology of HA in the current study has been addressed considering the variation in the driving force, such as supersaturation level (SSL), thermochemistry of the synthesized compounds.

\section{Materials and methods}

All the chemicals were of analytical grade and were used in their available form from commercial sources. Calcium chloride $(\geq 98 \%)$, TEA ( $\geq 97 \%)$, ortho-phosphoric acid ( $\geq 85 \%)$, ammonia $(\geq 25 \%)$ were purchased from Merck, India.

\section{Synthesis procedure}

Aliquot amount of the aqueous solution of calcium chloride $(0.1 \mathrm{M})$ was added into required amount of TEA in $25 \mathrm{ml}$ of water in order to ensure the complex formation. To the solution of calcium chloride in TEA was then added required amount of ortho-phosphoric acid $(0.06 \mathrm{M})$ to maintain the $\mathrm{Ca} / \mathrm{P}$ mole ratio at 1.67 . The $\mathrm{pH}$ of the resultant solution was adjusted to 8.5 with ammonium hydroxide solution to obtain a milky white suspension. This preparation procedure of the suspension was repeated by maintaining the solution temperature at 30 (room temperature), 50, 80 and $100{ }^{\circ} \mathrm{C}$. All the prepared suspensions were then separated through centrifugation and subsequently washed with water and dried under vacuum at $80{ }^{\circ} \mathrm{C}$ for $12 \mathrm{~h}$. The dried powders were ground, and were labeled as HAPRT, HAP50, HAP80 and HAP100 corresponding to the respective solution temperatures, i.e., room temperature, 50, 80 and $100{ }^{\circ} \mathrm{C}$ maintained for preparation of suspensions.

\section{Characterization}

Phase analysis of the prepared samples were carried out by X-ray diffraction (XRD) using Philips PW-1710 diffractometer $\mathrm{CuK} \alpha$ radiation $(\lambda=1.5418 \AA$ ) at a scan rate of $1.1^{\circ} \mathrm{min}^{-1}$ and with a sampling interval of $0.02(30 \mathrm{~mA}$ and $40 \mathrm{kV}$ ). The functional group analysis of the prepared samples were carried out using Fourier transformed infrared (FTIR) spectroscopy of model Perkin-Elmer Spectrum RXI instrument, within the scan range $4,000-450 \mathrm{~cm}^{-1}$. High-resolution transmission electron microscope (HRTEM) of JEOL (Model: JEM-2100 with an acceleration voltage $200 \mathrm{kV}$ ) was utilized for the morphological analysis of the synthesized HA samples. Measurement of electrophoretic mobility and conductivity was performed using Malvern Nanozeta analyzer.

\section{Results and discussion}

The phase analysis of the prepared HA samples were carried out by XRD and their respective diffractograms are represented in Fig. 1. The sharp XRD peaks in figure indicate that the prepared samples are highly crystalline. 


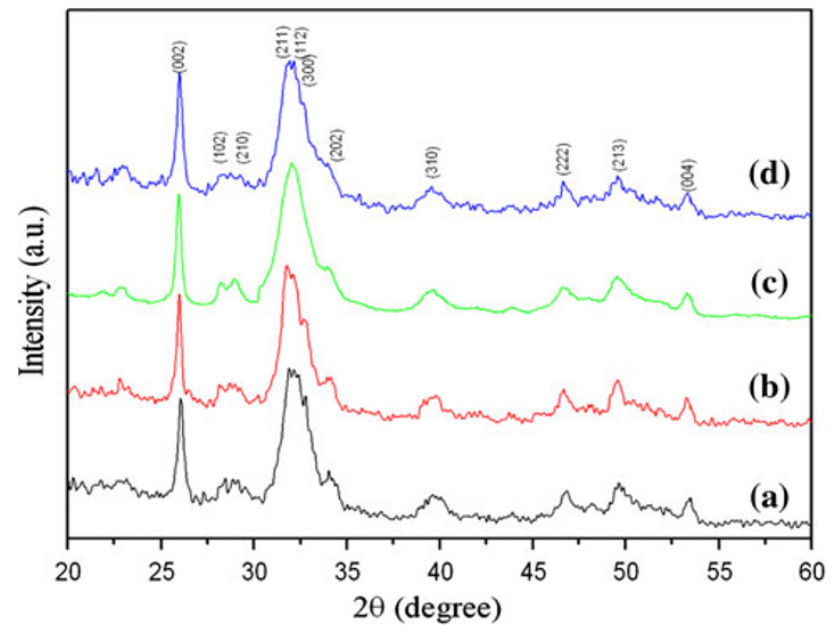

Fig. 1 X-ray powder diffraction patterns of a HAPRT, b HAP50, c HAP80 and d HAP100

Table 1 Summary of cell parameters calculated from XRD patterns of the synthesized hydroxyapatite samples

\begin{tabular}{llll}
\hline S. no. & Sample name & \multicolumn{2}{l}{ Lattice parameters } \\
\cline { 3 - 4 } & & $a=b(\AA)$ & $c(\AA)$ \\
\hline 1 & HAPRT & 9.4547 & 6.8365 \\
2 & HAP50 & 9.4481 & 6.8474 \\
3 & HAP80 & 9.4307 & 6.8546 \\
4 & HAP100 & 9.4298 & 6.8571 \\
\hline
\end{tabular}

The XRD patterns for all the synthesized samples are found to be well matched with that of standard HA material with hexagonal crystal structure and $\mathrm{P}_{3} / \mathrm{m}$ space group, reported in JCPDS data (card no. 09-0432). The lattice parameters $(a$ and $c$ ) of these samples are calculated by using the XRD data in the standard formula and are tabulated in Table 1. The calculated values of lattice parameter $a$ of the synthesized samples are found decreasing, while those of $c$ are found increasing with rise in preparation temperature. The relative intensities of the $\mathrm{d}_{002}$ peaks with respect to the major peaks $\left(\mathrm{d}_{211}\right)$ are estimated to be $74,80,82$ and $89 \%$ for the HAPRT, HAP50, HAP80 and HAP100 samples, respectively. The comparison of these values with that reported in standard JCPDS file no. 09-0432 for HA (33\%) reveals that the relative intensity of the $\mathrm{d}_{002}$ XRD peaks of the prepared samples gain a substantial increase. This observation indicates that crystal growth occurs along the [001] direction (i.e., along the $c$-axis) under the present preparation conditions (Zhang and $\mathrm{Lu}$ 2008).

The functional groups present in the prepared samples were investigated using Fourier transform infrared (FTIR) spectroscopy, which are shown in Fig. 2. The spectra reveals that all the prepared samples show the characteristic set of bands at 1,104, 1,064 and $1,028 \mathrm{~cm}^{-1}$

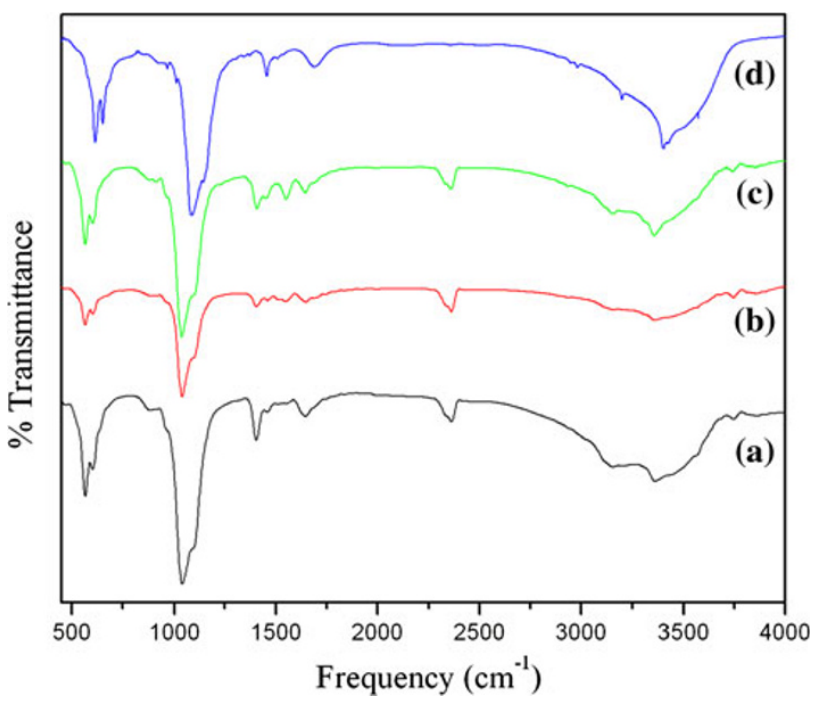

Fig. 2 FTIR spectra of HA samples a HAPRT, b HAP50, c HAP80 and d HAP100

corresponding to the triply degenerate asymmetric stretching mode of the $\mathrm{P}-\mathrm{O}$ bond $\left(v_{3}\right)$ in the apatite $\mathrm{PO}_{4}{ }^{3-}$ group. The band at $960 \mathrm{~cm}^{-1}$ can be assigned to the symmetric stretching mode of the $\mathrm{P}-\mathrm{O}$ bond $\left(v_{1}\right)$ (Puvvada et al. 2010). Additional bands at 604,578 and $563 \mathrm{~cm}^{-1}$ in the low wavenumber region represent the triply degenerated bending modes of the O-P-O bond $\left(v_{4}\right)$ (Puvvada et al. 2010; Sonju Clasen and Ruyter 1997). The observed peak in the region between 3,565 and $3,571 \mathrm{~cm}^{-1}$ and the weak band at $632 \mathrm{~cm}^{-1}$ can, respectively, be assigned to the stretching $\left(v_{\mathrm{s}}\right)$ and librational modes $\left(v_{\mathrm{L}}\right)$ of the structural hydroxyl anions in HA samples (Zhang and Lu 2008). The spectra also show two weak peaks at 2,866 and $2,980 \mathrm{~cm}^{-1}$, characteristic of $-\mathrm{CH}_{2}$ group, indicating the presence of TEA in the dried HA samples. From Fig. 2 it can be noted that the FTIR peaks for hydroxyl group in samples HAPRT, HAP50, HAP80 and HAP100 are, respectively, positioned at $3,565,3,568,3,570$, and $3,571 \mathrm{~cm}^{-1}$. It can also be noted that the peak position of hydroxyl group in HA samples are blue-shifted with respect to standard FTIR absorption value $\left(3,570 \mathrm{~cm}^{-1}\right)$ (Zhang and Lu 2008). This shift, which may have originated from the hydrogen bond between -OH group in TEA and $\mathrm{O}-\mathrm{H}$ at the surface of HA crystal, is higher for HAP$\mathrm{RT}$, and gradually decreases as the reaction temperature increases. Thus, this continuous shift of the FTIR peak towards higher wavenumber can be ascribed to the gradual disappearance of hydrogen bond between - $\mathrm{OH}$ group in TEA and $\mathrm{O}-\mathrm{H}$ at the surface of HA crystal with increase in the reaction temperature. This shift, in combination with the progressive enhancement of $-\mathrm{OH}$ stretching mode (at $3,571 \mathrm{~cm}^{-1}$ ) and librational mode (at $634 \mathrm{~cm}^{-1}$ ) indicates the disappearance of the interaction between the HA 
crystals and TEA (Zhang and Lu 2008). In other words, TEA desorbs slowly from the surface of HA particles into water owing to unstable surface adsorption due to increase in the reaction temperature. According to literature (Sonju Clasen and Ruyter 1997), carbonate ion substitution in HA crystal occurs in two ways, such as, A-type (when $\mathrm{CO}_{3}{ }^{2-}$ is substituted for $\mathrm{OH}^{-}$) and B-type (when $\mathrm{CO}_{3}{ }^{2-}$ is substituted for $\mathrm{PO}_{4}{ }^{3-}$ ). The appearance of twin peaks at 1,410 and $1,470 \mathrm{~cm}^{-1}$ (B-type) and the single peak at $874 \mathrm{~cm}^{-1}$ (A-type) in the present spectra (Fig. 2) suggests the presence of both type of carbonate ion substitutions in the synthesized $\mathrm{HA}$ samples. The required $\mathrm{CO}_{3}{ }^{2-}$ ions for these substitutions might have come from $\mathrm{CO}_{3}{ }^{2-}$ impurities in the reactants and/or from dissolved atmospheric $\mathrm{CO}_{2}$.

TEM studies were carried out for nanocrystalline HA samples and their representative micrographs are shown in Fig. 3. The micrographs reveal the formation of needleshaped morphology of the particles in the prepared samples. At room temperature, the prepared sample consists of needle-like particles with lengths in the range of $180-220 \mathrm{~nm}$. By increasing the temperature to $50^{\circ} \mathrm{C}$, particles retained similar morphology but had an increased particle size (lengths in the range of 185-235 nm). We have further increased the temperature to $80^{\circ} \mathrm{C}$, and observed that the morphologies of some of the needle-shaped particles have started to change into spherical shape (Fig. 3c) and the lengths of the needle-shape particles are reduced to $65-85 \mathrm{~nm}$. Finally, when the temperature is increased to $100{ }^{\circ} \mathrm{C}$, only spherical particles are formed, as is indicated in Fig. 3d. Asaoka et al. (1995) have also observed similar morphological changes for HA samples when they varied the reaction temperature for the preparation of the samples. It is believed that the growth of HA particles along $c$-axis is facilitated by the effective interaction of TEA molecules with the surfaces of the crystals. So the change in size and shape of the HA particles at different temperature can be attributed to the different degree of interaction of TEA molecule with HA crystal, which is driven by temperature. FTIR spectral analysis also infers that there is variation in interaction of TEA molecules on the surfaces of crystals with change in temperature.

Figure 4 shows a representative HRTEM image of needle-like HA nanoparticles, which clearly displayed the lattices planes with spacing of 0.324 and $0.798 \mathrm{~nm}$ corresponding to the d-spacing of the (002) and (100) planes of hexagonal phase of HA, respectively. The Fast Fourier transform (FFT) pattern (inset) obtained from this figure showed the planes, which could be identified as hexagonal crystal structure of HA. The (100) plane is found to be parallel to the growth direction, suggesting that nanoparticles prefer to grow along the [001] direction.
Fig. 3 TEM images of HA nanoparticles prepared at different temperatures a HAPRT, b HAP50 and c HAP80 and d HAP100
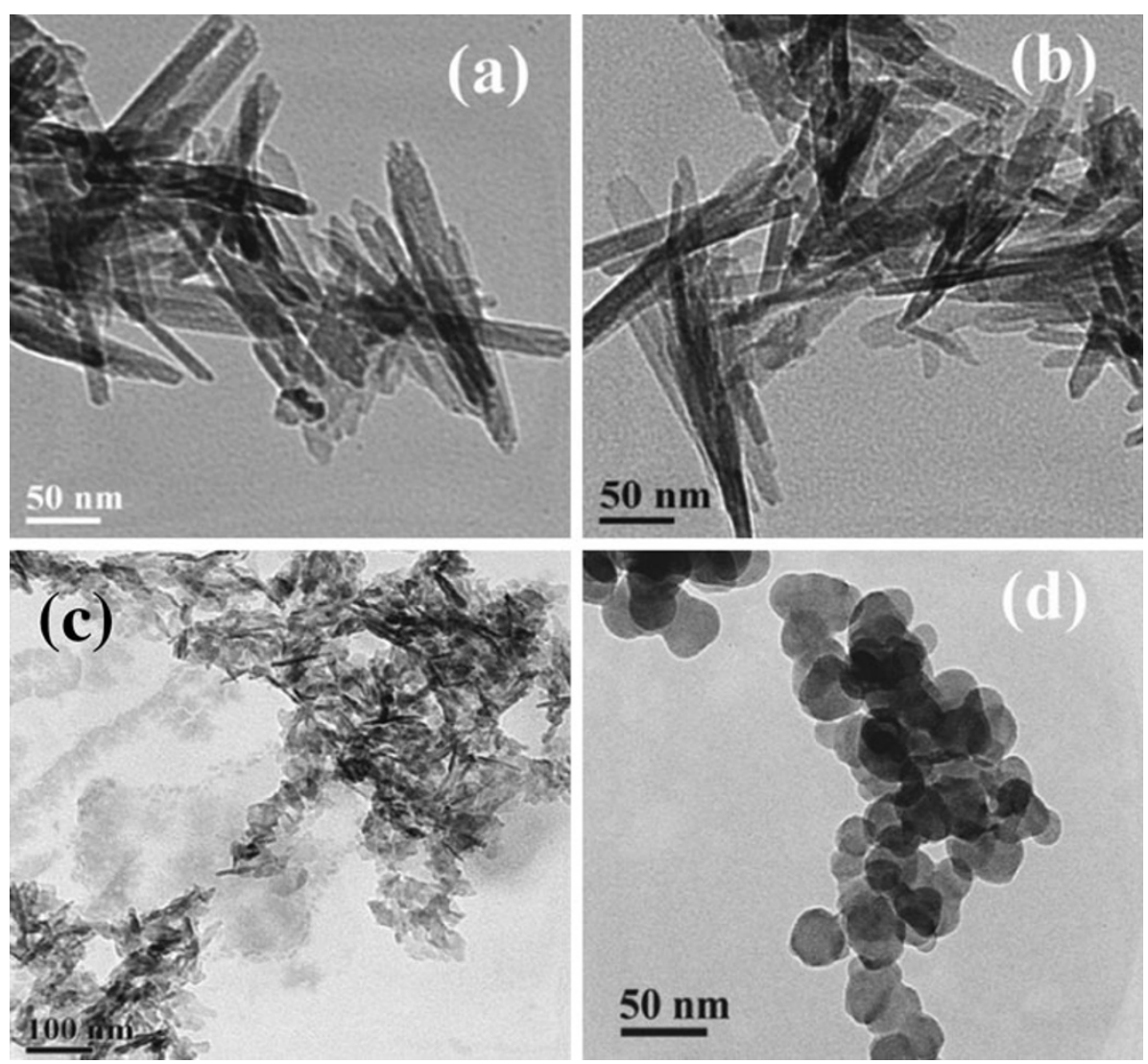


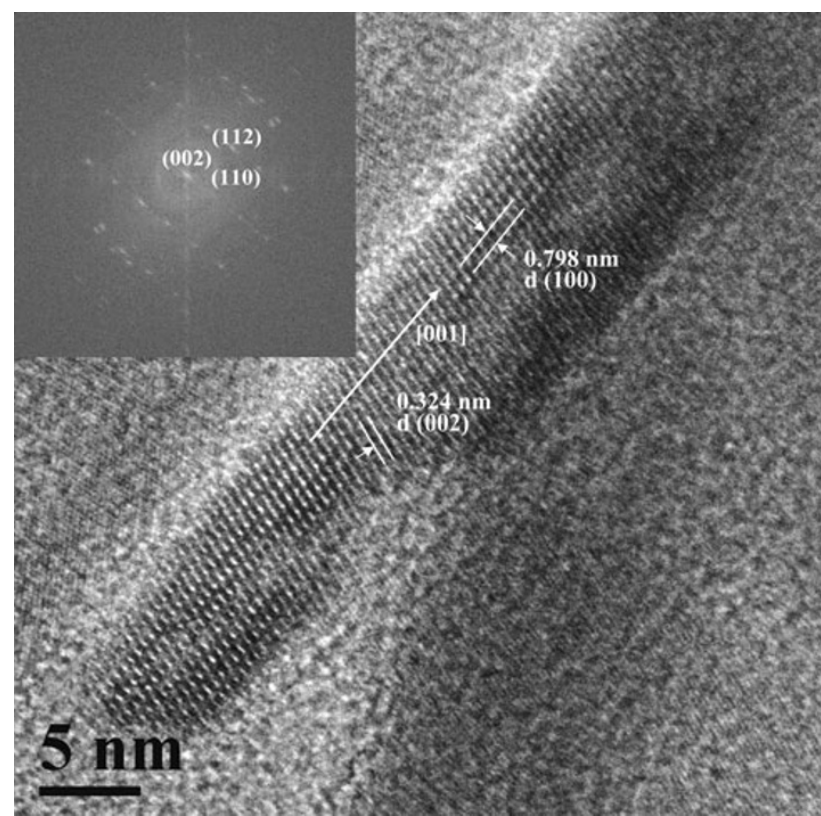

Fig. 4 HRTEM image of HA prepared at a temperature of $50{ }^{\circ} \mathrm{C}$ and FFT image of corresponding $\mathrm{HA}$ as an inset

Based on the literature, the possible cause for the temperature-dependent variation in the morphology of the resultant nanoparticles, prepared through chemical precipitation method, may be the difference in the precipitation rate of HA from the reaction mixture. The precipitation reaction for the formation of HA can be expressed by the Eq. 1.

$10 \mathrm{Ca}^{2+}+6 \mathrm{PO}_{4}{ }^{3-}+2 \mathrm{OH}^{-} \rightarrow \mathrm{Ca}_{10}\left(\mathrm{PO}_{4}\right)_{6}(\mathrm{OH})_{2}$

The precipitation rate of $\mathrm{HA}$ is, however, governed by a thermodynamic relationship, as given by Eq. 2 .

$\Delta G_{\mathrm{HA}}=-\frac{R T}{v} \ln \frac{x}{k_{\mathrm{sp}}}$

where, $\Delta G_{\mathrm{HA}}$ is the difference in Gibbs free energy between the supersaturated and equilibrium solution of HA at a given temperature. The other terms in the equation, such as $T, k_{\mathrm{sp}}$ and $x$ represent various driving forces, such as absolute temperature, solubility product and ionization (or ionic activity) product, respectively, of HA. The symbols $v$ and $R$ are the number of ions and the universal gas constant $(8.314 \mathrm{~J} / \mathrm{mol} \mathrm{K})$.

The ionic activity product $(x)$ depends on the activity of the ions and can be expressed as follows (Eq. 3):

$x=\left[a_{\mathrm{Ca}^{2+}}\right]^{5}\left[a_{\mathrm{PO}_{4}^{3-}}\right]^{3}\left[a_{\mathrm{OH}^{-}}\right]$.

In this equation (3), the terms in the square bracket represent activity of the subscripted ionic species, where the activity $(a)$ is known to be equal to the product of the concentrations of ions $(C)$ and the activity coefficient $(\gamma)$.
According to this equation, the value of $v$ for the synthesis of HA is equal to 9. From Eq. 2, it can be inferred that $\Delta G_{\mathrm{HA}}$ increases with increasing temperature $(T)$ and $x$, and with decreasing $k_{\mathrm{sp}}$ (Kumar et al. 2004). As a consequence, a greater driving force for precipitation, and hence, a faster precipitation rate is expected with increasing temperature and decreasing $k_{\mathrm{sp}}$ of HA for a given $x$. It has been experimentally verified that the $k_{\mathrm{sp}}$ of $\mathrm{HA}$ samples decreases with increasing temperature (Kumar et al. 2004; Prakash et al. 2006a, b), and the relationship between $k_{\text {sp }}$ and $T$ can be expressed by Eq. 4 .

$\log k_{\mathrm{sp}}=-\frac{p}{T}-q-r T$

Here, $p, q$ and $r$ are constants and equal to 8,219.416, 1.66572 and 0.0981 , respectively. Using Eq. 4, the solubility product of HA in the present study is estimated and it is found that the value of $k_{\mathrm{sp}}$ decreases from $2.34 \times 10^{-59}$ to $1.458 \times 10^{-59}$ as the temperature increases from room temperature to $50{ }^{\circ} \mathrm{C}$, it further drops to $2.398 \times 10^{-60}$ as the temperature reaches to $80{ }^{\circ} \mathrm{C}$, and finally drops to $4.619 \times 10^{-61}$ as the temperature reaches $100{ }^{\circ} \mathrm{C}$. However, as per the report by Badillo-Almaraz and Ly (2003) the dissolution of HA gets facilitated by decreasing the $\mathrm{pH}$ and the $\mathrm{Ca}^{2+}$ ion concentration at a certain temperature. They have verified this fact from the solubility isotherms, a function of $\mathrm{pH}$ at various $\mathrm{Ca}^{2+}$ ion concentrations for $\mathrm{HA}$ at $25^{\circ} \mathrm{C}$. Similarly, according to Ito et al. (1997), the solubility of HA increases in solutions containing carbonate ions, and the $k_{\mathrm{sp}}$ of carbonated-apatite becomes greater than that of HA. As suggested from FTIR spectral analysis, the prepared HA samples contain both Aand B-type carbonate substitution and the carbonate ion concentrations in the samples decrease with increasing temperature (which was analyzed on the basis of A-type and B-type peak intensities compared to maximum intense peak in each synthesized sample). Hence, it is expected that the concentration of carbonate ions in solution may increase with temperature.

The solubility product estimated from Eq. 4 cannot be used to measure the supersaturation level (SSL) at any given temperature because of the following reasons: first, the applicability of Eq. 4 is doubtful at high temperatures, second reason is the lack of sufficient solubility data of HA in the temperature range used in this study, and third cause is dependence of solubility/solubility product of $\mathrm{HA}$ on the concentration of $\mathrm{Ca}^{2+}$ and $\mathrm{CO}_{3}{ }^{2-}$ ions, $\mathrm{pH}$ as well as the composition and particle size. The ionic product in Eq. 2, however, may not be affected much by temperature, as the activity of $\mathrm{Ca}^{2+}$ ions does not decrease appreciably with increasing temperature for completely soluble salts. According to Eqs. 2 and 4, a decrease in precipitation rate with increasing temperature is thus expected in the case, 
Fig. 5 Electrophoretic mobility of HA samples prepared at various temperatures: a HAPRT, b HAP50, c HAP80 and d HAP100

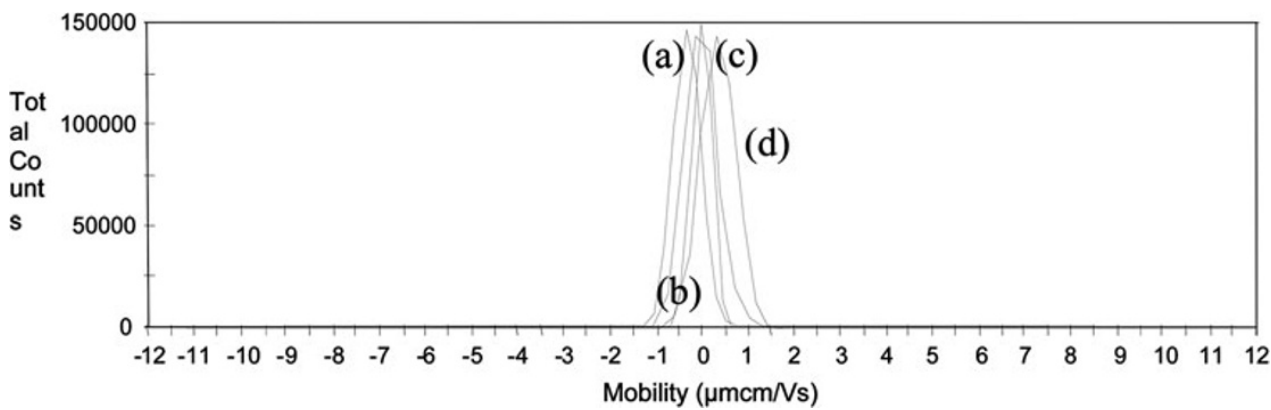

whereby the particle size of the HA samples decreases with increasing temperature and therefore, it deserves attention and analysis.

$x=k_{\mathrm{sp}}(\mathrm{SSL}+1)^{9}$

Furthermore, computation of the SSL and $\Delta G_{\mathrm{HA}}$ is very complicated at different stages of formation of $\mathrm{HA}$ compounds because of the effect of the parameters, such as $\mathrm{Ca}^{2+}$ ion concentration, $\mathrm{pH}, \mathrm{CO}_{3}{ }^{2-}$ ion concentration, etc., on the apparent solubility of HA. Here, $\mathrm{CO}_{3}{ }^{2-}$ ion concentration decreases with increase in temperature, which favors the dissolution of HA. It may be possible that the apparent solubility of HA could have a positive dependence on temperature. Previous literature suggests that the apparent solubility of HA increases with increase in temperature, and thus from Eq. 5, the SSL decreases (for completely soluble salts). However, $\Delta G_{\mathrm{HA}}$ does not change significantly, because the driving force increases with the temperature and decreases with the logarithmic term of Eq. 2. The increase in $\Delta G_{\mathrm{HA}}$ is about 1.07 times, when the temperature is increased from room temperature to $50{ }^{\circ} \mathrm{C}$. This value further increases to 1.175 times, when the temperature is raised to $80{ }^{\circ} \mathrm{C}$, and to 1.19 times, when the temperature is increased to $100{ }^{\circ} \mathrm{C}$ for a given $x / k_{\mathrm{sp}}$ ratio. However, the value of $\Delta G_{\mathrm{HA}}$ decreases about 2.30 times, when the $x / k_{\mathrm{sp}}$ ratio is decreased 1 order of magnitude at a given temperature. Therefore, the decrease in SSL results in a net slower growth rate as temperature is increased. It is likely that this effect is manifested in the observed morphological change of the particles with temperature (Fig. 3).

In fact, the $\mathrm{CO}_{3}{ }^{2-}$ ion concentration decreases, when the HA sample is prepared with TEA at various temperatures. The presence of librational mode peaks at this temperature (Fig. 2) indicates the disappearance of interaction with HA crystal during this process. TEA can be desorbed into water because of the unstable surface adsorption (Zhang and Lu 2008). Thus, it can be concluded that the disappearance of interaction with temperature plays a major role in altering the morphology of the crystallites. In other words, the thermochemistry of this reaction also plays a major role in affecting the particle size.
The standard heat of formation $\left(\Delta H_{\mathrm{f}}\right)$ of various compounds and ions, such as $\mathrm{CaCl}_{2}, \mathrm{H}_{3} \mathrm{PO}_{4}$, TEA, $\mathrm{Ca}_{10}\left(\mathrm{PO}_{4}\right)_{6}(\mathrm{OH})_{2}, \mathrm{HCl}, \mathrm{H}_{2} \mathrm{O}$ and $\mathrm{OH}^{-}$are $-795.8,-1294.1$, $-664.2,-13399.0,-166.6,-285.8$ and $-229.9 \mathrm{~kJ} / \mathrm{mol}$, respectively (Kumar et al. 2004; Prakash et al. 2006a, b; Masterton et al. 1987). By using these reported data, the heat of reaction for the balanced reaction in Eq. 6 has been calculated, and is found to be $-326.8 \mathrm{~kJ} / \mathrm{mol}$. The negative sign infers that this reaction is exothermic.

$$
\begin{gathered}
10 \mathrm{CaCl}_{2}+6 \mathrm{H}_{3} \mathrm{PO}_{4} \underset{-\mathrm{TEA}}{\stackrel{4 \mathrm{OH}^{-}+\mathrm{TEA}}{\longrightarrow}} \mathrm{Ca}_{10}\left(\mathrm{PO}_{4}\right)_{6}(\mathrm{OH})_{2}+18 \mathrm{HCl} \\
+2 \mathrm{H}_{2} \mathrm{O}
\end{gathered}
$$

Thus, according to the Le Chatelier's principle, the equilibrium position in reaction 6 will shift towards the reactants side, if the reaction temperature is increased. In order to verify this, the end point conductivity of the prepared samples has been measured through zetasizer, and are found to be 0.0628 (HAP100), 0.0547 (HAP80), 0.0202 (HAP50) and 0.0160 (HAPRT), $\mathrm{mS} / \mathrm{cm}$. The order of end point conductivities of all the prepared samples is: HAP100 $>$ HAP80 $>$ HAP50 $>$ HAPRT. This finding is supported by increase in electrophoretic mobility with increase in temperature as shown in Fig. 5. The increase in conductivity of the reaction 6 with increasing temperature reveals an enhancement in the apparent solubility of HA (Prakash et al. 2006a, b).

\section{Conclusions}

In summary, HA nanoparticles have been synthesized at different temperatures through reaction between calcium chloride, suitably complexed with TEA, and ortho-phosphoric acid. The phase of the HA samples have been studied by XRD and FTIR spectroscopy. The particle size and morphologies of the prepared nanoparticles changes from needle shape to spherical by increasing the reaction temperature from room temperature $\left(30{ }^{\circ} \mathrm{C}\right)$ to $100{ }^{\circ} \mathrm{C}$. This change in morphologies of the synthesized HA samples with respect to temperature is attributed to the 
different precipitation rate and interaction of TEA molecules with the surfaces of the HA crystals. This prediction has been verified through end point conductivity as well as electrophoretic mobility measurement and supported by the thermochemistry of this present reaction. The present study has provided the procedure to obtain HA nanoparticles with controlled morphology and size in view of its size and shape-dependent biological applications, such as drug delivery, removal of toxic elements and various mechanical properties, etc.

Open Access This article is distributed under the terms of the Creative Commons Attribution License which permits any use, distribution, and reproduction in any medium, provided the original author(s) and the source are credited.

\section{References}

Asaoka N, Best S, Knowles JC, Bonfield W (1995) Characterization of hydroxyapatite precipitated from different reactants. Bioceramics 8:331

Badillo-Almaraz VE, Ly J (2003) Calcium sorption on hydroxyapatite in aqueous solutions: reversible and nonreversible components. J Colloid Interface Sci 258:27

Bogdanoviciene I, Beganskiene A, Tonsuaadu K, Glaser J, Meyer HJ, Kareiva A (2006) Calcium hydroxyapatite, $\mathrm{Ca}_{10}\left(\mathrm{PO}_{4}\right)_{6}(\mathrm{OH})_{2}$ ceramics prepared by aqueous sol-gel processing. Mater Res Bull 41:1754

Earl JS, Wood DJ, Mile SJ (2006) Hydrothermal synthesis of hydroxyapatite. J Phys Conf 26:268

Glenn H (2008) Imaging of metabolic bone diseases. Best Pract Res Clin Rheumatol 22:1127

Hurson S, Lacefield W, Lucas L, Ong J, Whitehead R, Bumgardner J (1993) Transactions of the 19th annual meeting of the society for biomaterials, (1993) Birmingham, AL, April 28-May 2, pp 223

Ito A, Maekawa K, Tsutsumi S, Ikazaki F, Tateishi T (1997) Solubility product of $\mathrm{OH}$-carbonated hydroxyapatite. J Biomed Mater Res 36:522

Kumar R, Prakash KH, Cheang P, Khor KA (2004) Temperature driven morphological changes of chemically precipitated hydroxyapatite nanoparticles. Langmuir 20:5196

LeGeros RZ (1988) Calcium phosphate materials in restorative dentistry: a review. Adv Dent Res 2:164

Lim GK, Wang J, Ng SC, Chew CH, Ganl LM (1997) Processing of hydroxyapatite via microemulsion and emulsion routes. Biomaterials 18:1433
Luo P, Nieh TG (1996) Preparing hydroxyapatite powders with controlled morphology. Biomaterials 17:1959

Masterton WL, Slowinski EJ, Stanitski CL (1987) Chemical principles, 6th edn. CBS College Publishing, San Francisco

Prakash KH, Kumar R, Ooi CP, Cheang P, Khor KA (2006a) Apparent solubility of hydroxyapatite in aqueous medium and its influence on the morphology of nanocrystallites with precipitation temperature. Langmuir 22:11002

Prakash KH, Ooil CP, Kumar R, Khor KA, Cheang P (2006) IEEE conference on emerging technologies-Nanoelectronics, Singapore, 10-13 Jan 2006, pp 345-349

Puvvada N, Panigrahi PK, Pathak A (2010) Room temperature synthesis of highly hemocompatible hydroxyapatite, study of their physical properties and spectroscopic correlation of particle size. Nanoscale 2:2631

Puvvada N, Panigrahi PK, Mandal D, Pathak A (2012) Shape dependent peroxidase mimetic activity towards oxidation of pyrogallol by $\mathrm{H}_{2} \mathrm{O}_{2}$. RSC Adv 2:3270

Ramachandra R, Roopa HN, Kannan TS (1997) Solid state synthesis and thermal stability of HAP and HAP- $\beta$-TCP composite ceramic powders. J Mater Sci Mater Med 8:511

Shirkhanzadeh M (1998) Direct formation of nanophase hydroxyapatite on cathodically polarized electrodes. J Mater Sci Mater Med 9:67

Sonju Clasen AB, Ruyter IE (1997) Quantitative determination of type $\mathrm{A}$ and type $\mathrm{B}$ carbonate in human deciduous and permanent enamel by means of Fourier transform infrared spectrometry. Adv Dent Res 11:523

Uota M, Arakawa H, Kitamura N, Yoshimura T, Tanaka J, Kijima T (2005) Synthesis of high surface area hydroxyapatite nanoparticles by mixed surfactant-mediated approach. Langmuir 21:4724

Xia W, Chang J (2006) Well-ordered mesoporous bioactive glasses (MBG): a promising bioactive drug delivery system. J Control Release 110:522

Xu Z, Liu C, Wei J, Sun J (2012) Effects of four types of hydroxyapatite nanoparticles with different nanocrystal morphologies and sizes on apoptosis in rat osteoblasts. J Appl Toxicol 32:429

Yamashita Y, Uchida A, Yamakawa T, Shinto Y, Araki N, Kato K (1998) Treatment of chronic osteomyelitis using calcium hydroxyapatite ceramic implants impregnated with antibiotic. Int Orthop 22:247

Yang Q, Wang SH, Fan PW, Wang LF, Di Y, Lin KF, Xiao FS (2005) $\mathrm{pH}$-Responsive carrier system based on carboxylic acid modified mesoporous silica and polyelectrolyte for drug delivery. Chem Mater 17:5999

Zhang Y, Lu JA (2008) A mild and efficient biomimetic synthesis of rodlike hydroxyapatite particles with a high aspect ratio using polyvinylpyrrolidone as capping agent. Cryst Growth Des $8: 2101$ 